\title{
PROTOCOLO ELETRÔNICO MULTIPROFISSIONAL EM CIRURGIA BARIÁTRICA*
}

\author{
Multiprofessional electronic protocol in bariatric surgery* \\ Denise Serpa Bopp NASSIF, Osvaldo MALAFAIA, Paulo Afonso Nunes NASSIF, \\ Carlos Henrique KURETZKI, Ricardo Wallace das Chagas LUCAS, José Simão de Paula PINTO
}

Trabalho realizado no Programa e PósGraduação em Clínica Cirúrgica da Universidade Federal do Paraná, Curitiba, PR, Brasil.

DESCRITORES - Obesidade mórbida. Protocolos eletrônicos. Informática médica.
RESUMO - Racional - A obesidade é doença multifatorial. A coleta de dados exerce papel fundamental para realização de pesquisas de alto grau de qualidadee, na atualidade, a cirurgia da obesidade carece de meios para realização de pesquisas prospectivas com elevado nível de confiabilidade. Os dados obtidos em protocolos eletrônicos são mais completos, têm poucos erros, são mais consistentes e apresentam baixo percentual de violação em relação aos prontuários de papel. Objetivo - 1) Criar base teórica de dados clínicos referentes à multiprofissionalidade na abrangência da Cirurgia Bariátrica e Metabólica; 2) informatizar essa base transformando-a em protocolo eletrônico multiprofissional; 3) incorporá-la ao SINPE ${ }^{\circ}$; 4) disponibilizar este protocolo eletrônico e suas coletas para visualização via internet utilizando SINPE WEB ${ }^{\circ}$. Método - A criação da base teórica em Word ${ }^{\odot}$ - depois transformada em forma digital para ser utilizada como protocolo eletrônico - foi baseada na revisão bibliográfica de nove livros-texto em obesidade mórbida, cirurgia bariátrica e metabólica, livros de multiprofissionalidade, livros específicos em psicologia e nutrição aplicados ao tema. Adicionou-se revisão sistemática da literatura atual com base em artigos científicos publicados nos últimos cinco anos no Medline/Pubmed, Lilacs e Scielo e também em teses e dissertações realizadas com protocolos eletrônicos. Assim, foram informatizados 10.306 itens de forma hierarquizada e ramificada contendo dados relacionados à medicina, fisioterapia, psicologia e nutrição. Resultados - O protocolo completo poderá ser acessado para visualização no site www.sinpe.com.br. Conclusões - 1) Foi possível criar base eletrônica de dados clínicos referentes à multiprofissionalidade em cirurgia bariátrica e metabólica para coleta padronizada de informações; 2) foi possível informatizar esta base de dados; 3) ela está incorporada ao SINPE ; 4) o protocolo eletrônico e suas coletas foram disponibilizados para visualização via internet utilizando SINPE WEB ${ }^{\circ}$.

ABSTRACT - Background - Obesity is a multi factorial disease. Data collection plays a fundamental role for conducting papers with high level of quality and obesity surgery lacks the means to carry out prospective studies with high reliability. Data from electronic protocols are more complete, have fewer errors, are more consistent and have a low percentage of violations in relation to paper charts. Aim - 1) To create a theoretical basis of clinical data regarding the multiprofessionalism on metabolic and bariatric surgery; 2) to computerize this basis by transforming it into electronic multi professional protocol; 3) to incorporate it into the SINPE ${ }^{\odot}$; 4) to provide the protocol address and their collections for viewing via the Internet using SINPE WEB ${ }^{\circ}$. Method - The creation of the theoretical background was done in Word $^{\circ}$ - later transformed into digital form to be used as electronic protocol - based on literature review of nine textbooks in morbid obesity, bariatric surgery and metabolic multi professionalism books, special books in psychology and nutrition applied to the subject. Was added systematic review of the literature based on scientific articles published over the last five years in Medline/Pubmed, Lilacs and Scielo and in theses and dissertations done with electronic protocols. Thus, 10,306 items were computerized in a hierarchical and branched containing data related to medicine, physiotherapy, psychology and nutrition applied to the subject. Results - The complete protocol can be accessed to view in site www.sinpe.com.br - Conclusions - 1) It was possible to create electronic database regarding the clinical multi professionalism on bariatric and metabolic surgery to collect standardized information; 2) it was possible to computerized the database; 3 ) it is incorporated into the SINPE ${ }^{\circ}$; 4 ) the electronic protocol and its collections were made available for viewing via the Internet using SINPE WEB ${ }^{\circ}$.
HEADINGS - Obesity, morbid. Eletronic protocols. Medical informatics. 


\section{INTRODUÇÃO}

A obesidade é doença crônica multifatorial, comumente associada à complicações. Quando ndicado tratamento cirúrgico, faz-se necessário que a condução do procedimento seja realizado por equipe multidisciplinar e o seu resultado estÁ diretamente ligado à mudanças de hábitos de vida 5 .

É fundamental, portanto, que cada um dos integrantes da equipe multidisciplinar tenha visão geral composta pelos mais diversos dados coletados no diagnóstico. Nesse sentido, a possibilidade de utilização de protocolos eletrônicos proporciona a manutenção das informações de forma confiável, aperfeiçoa o armazenamento e a análise dos dados, por meio de uma matriz digitalizada para que possa ser acessada a qualquer tempo ${ }^{13}$.

A qualidade dos dados obtidos dos protocolos eletrônicos é mais completa, tem poucos erros, é mais consistente e apresenta baixo percentual de violação em relação aos prontuários de papel ${ }^{4}$.

Colaborando com a área de informática médica, e visando oferecer alternativa para a elaboração de protocolos, foi criada pelo Prof. Dr. Osvaldo Malafaia software que originou a linha de pesquisa no Programa de Pós-Graduação em Clínica Cirúrgica da Universidade Federal do Paraná. Com arquitetura identificada por meio da sigla SINPE ${ }^{\odot}$ - Sistema Integrado de Protocolos Eletrônicos - a idéia do que viria a tornar-se o SINPE ${ }^{\odot}$ surgiu em 1992, quando ele sugeriu a criação de sua linha de pesquisa em Protocolos Eletrônicos em Cirurgia. O SINPE ${ }^{\odot}$ tem registro de propriedade intelectual no INPI, sob número RS 06056-1.

O SINPE ${ }^{\odot}$ foi desenvolvido visando solucionar algumas deficiências tipicamente encontradas em pesquisa, tais como a falta de padronização dos termos, a não utilização dos dados de uma pesquisa em outra, dificuldades para realização de pesquisa multicêntrica e também multidisciplinar ${ }^{6}$.

Existem atualmente protocolos eletrônicos nas áreas de cirurgia do aparelho digestivo, urologia, oftalmologia, enfermagem, fisioterapia e gestão já desenvolvidos e incorporados ao SINPE $^{\circ}$ e outros protocolos estão em desenvolvimento. Desde a sua criação a área da saúde avançou na coleta e armazenamento de dados científicos, tornando o acesso e o cruzamento dessas informações de maneira rápida e segura, em uma importante ferramenta para o crescimento do universo científico e da pesquisa ${ }^{12}$.

Este trabalho tem por objetivos: 1) criar base teórica de dados clínicos referentes à multiprofissionalidade na abrangência da cirurgia bariátrica e metabólica; 2) informatizar essa base transformando-a em protocolo eletrônico multiprofissional; 3) incorporá-la ao SINPE ${ }^{\odot}$; 4) disponibilizar este protocolo eletrônico e suas coletas para visualização via internet utilizando SINPE WEB ${ }^{\circ}$.

MÉTODO

O objetivo da primeira etapa foi criar uma base teórica de dados clínicos referentes à atuação da medicina, nutrição, psicologia e fisioterapia para futura inclusão no protocolo eletrônico informatizado.

A revisão bibliográfica foi realizada com a escolha de nove livros-texto devidamente reconhecidos em obesidade mórbida e cirurgia bariátrica e metabólica, livros de multiprofissionalidade, livros específicos em psicologia e nutrição aplicados à obesidade mórbida e cirurgia bariátrica e metabólica. Foram eles:

- Update: Surgery for the Morbidly Obese Patient (Deitel; Cowan Jr., 2000);

- Cuidados Pré e Pós-operatórios na Cirurgia da Obesidade (Kawahara, 2005);

- Cirurgia da Obesidade (Garrido Jr. et al, 2002);

- Síndrome Metabólica: Conceitos Atuais. (Luna, 2006);

- Uma Abordagem Multidisciplinar. (Lancha Jr., 2006);

- Obesidade: Perguntas e Respostas (Dâmaso; TOCK, 2005);

- Obesity Surgery - Principles and Practices (Pitombo et al. 2008);

- Transtornos Alimentares e Obesidade (Claudino; Zanella, 2005);

- Contribuições da Psicologia na Cirurgia da Obesidade (Franques; Arenalis-Loli, 2006).

A avaliação pré-operatória da medicina foi realizada através dos principais exames solicitados em vários serviços de cirurgia bariátrica, com auxílio de laboratório de análises clínicas, visitas e orientações dos médicos de cada especialidade, ou seja, cardiologista, pneumologista, gastroenterologista, endocrinologistas e endoscopista.

A avaliação da fisioterapia foi baseada no protocolo de atendimento utilizado no Serviço de Cirurgia Bariátrica e Metabólica do Hospital Universitário Evangélico de Curitiba e Hospital Nossa Senhora do Rocio de Campo Largo, PR, Brasil.

Para a formação dos itens da nutrição e psicologia, existiu a necessidade da busca de profissionais da área, para auxiliar o levantamento bibliográfico. No caso da nutrição, o protocolo foi baseado no utilizado pela Pontifícia Universidade Católica do Rio Grande do Sul. A psicologia também teve participação de duas profissionais de referência da área; após consenso entre a literatura e a atuação destas profissionais foram criados os itens e suas ramificações.

Após o estudo dos referidos livros iniciou-se revisão sistemática da literatura atual com base em artigos científicos publicados nos últimos cinco anos, que foram coletados em três bases de pesquisa eletrônica diferentes: Medline/Pubmed, Lilacs e Scielo e também em teses e dissertações realizadas com protocolos eletrônicos. Alguns trabalhos anteriores ao período citado foram consultados quando relevantes.

\section{Manuseio do software}

Após a revisão da literatura nos livros-texto e nos artigos na internet, foram levantados 10.306 itens, agrupados em seis segmentos principais: Anamnese; Exame Físico; Avaliação Pré-operatória; Resultados da 
Avaliação Pré e Pós operatória; Tratamentos e Evolução. Cada segmento gerou outras ramificações, criadas na forma de árvore.

Para melhor entendimento e acompanhamento eletrônico do que será exposto é necessário que o leitor navegue no site www.sinpe.com.br onde encontrará para visualização todo o protocolo criado e os casos já incorporados envolvendo a cirurgia bariátrica e metabólica.

\section{RESULTADOS}

Para compreensão inicial do protocolo, ele é dividido em duas grandes partes: o protocolo mestre e o(s) específico(s). O protocolo mestre contém 10.306 itens com todas as informações (itens) relacionados ao tema e em sua multiprofissionalidade. O específico, é parte deste mestre e contém os itens resgatados do mestre e diretamente relacionados à pesquisa, mais ou menos ampla que se queira fazer - qualquer que ela seja -, desde que as informações tenham sido colhidas e armazenadas no protocolo mestre.

A seguir será feito tutorial de como navegar no software quando aberto para visualização.

1) Acesse no endereço <http://www.sinpe.com.br/ sinpe/> o Protocolo Multiprofissional de Cirurgia Bariátrica contendo as coletas realizadas. Para visualizar este protocolo pelo SINPE WEB ${ }^{\circ}$ use login usuário "visitante", senha "visitante" e instituição HUEC (Figura 1).

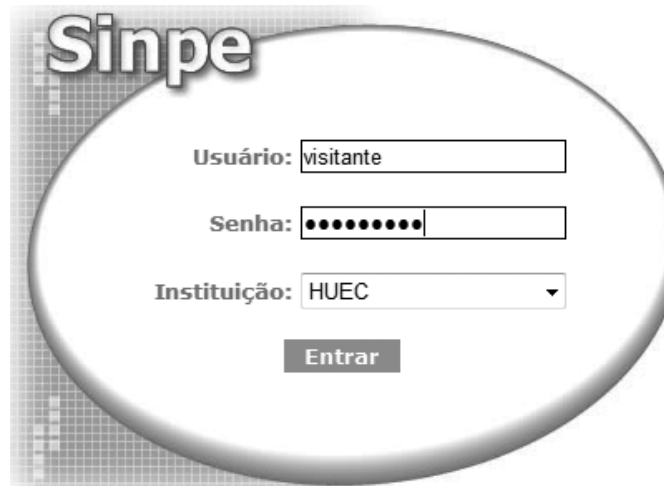

FIGURA 1 - Tela inicial para acesso ao SINPE WEB ${ }^{\circ}$

2) Após clicar no botão "Entrar" aparecerá a tela abaixo, solicitando a seleção do protocolo mestre. Clique no botão "Continuar"(Figura 2).

\section{Sinpe}

:Seleção de protocolo mestre :.

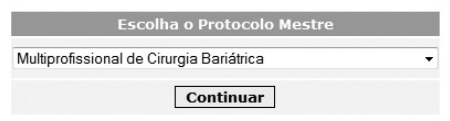

FIGURA 2 - Seleção do protocolo mestre para visualização
3) O usuário terá permissão de visualização deste protocolo não lhe sendo permitida alteração de qualquer dado registrado no programa (Figura 3).

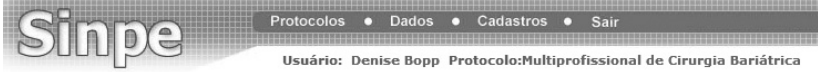

FIGURA 3 - Tela principal da aplicação SINPE WEB ${ }^{\circ}$

4) As informações do protocolo mestre são apresentadas através da opção do menu "Protocolos" e "Mestre" (Figura 4).
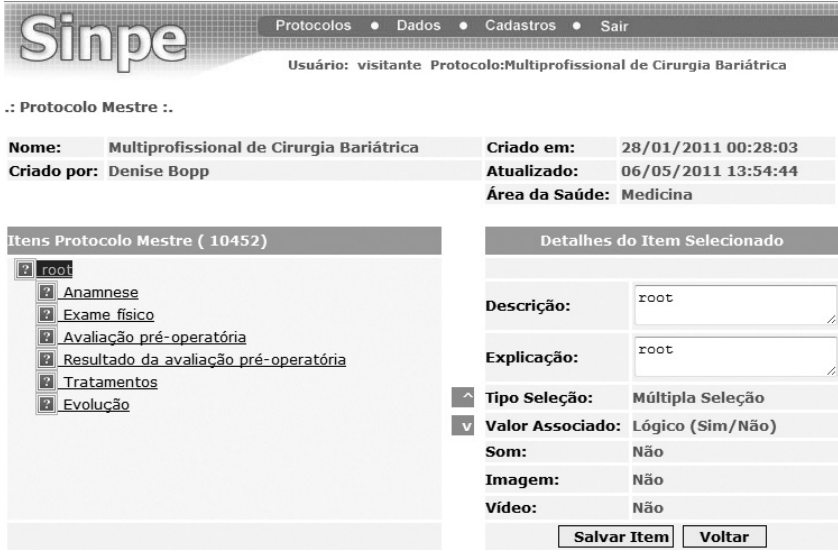

FIGURA 4 - Tela contendo o protocolo mestre para visualização

5) Para visualizar as coletas efetuadas abra a opção "Dados" e "Coletar" (Figura 5).

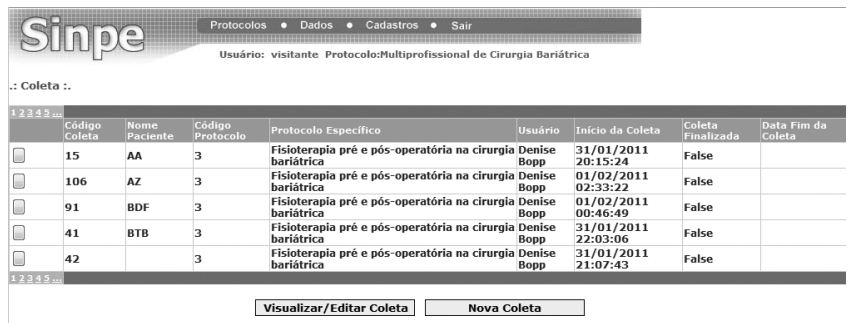

FIGURA 5 - Tela contendo as coletas para visualização

6) O programa define o tipo de usuário que acessa o sistema, bem como a permissão outorgada a ele. Os tipos de usuários são: super-usuário (pode criar outros usuários, cadastrar instituições, bem como atribuir permissões a outros usuários, tem acesso irrestrito a manipulação de outros protocolos); criador de protocolos (pode criar novos protocolos, tendo permissão administrativa sobre os mesmos); usuário comum (só pode utilizar o sistema mediante permissão outorgada).

7) Com relação às permissões, elas podem ser de quatro tipos: administrador (possui todos os direitos sobre os protocolos mestre e específico, podendo 
incluir, excluir ou alterar dados); coletor (tem permissão para cadastrar pacientes e coletar dados somente); visualizador (pode consultar a estrutura dos protocolos mestre e específicos sem realizar alterações); pesquisador (tem possibilidade apenas de realizar pesquisa nos dados coletados).

8) Para manuseio de dados clínicos inicia-se pelo protocolo mestre (conjunto de dados dispostos de forma hierarquizada, denominadas de pastas, as quais são subdivididas em itens e subitens, distribuídos por diferentes gerações denominadas "irmão" e "filho", que definem a formatação do conteúdo da base teórica. Posteriormente, realiza-se a confecção dos protocolos específicos criados a partir do protocolo mestre. Para exemplo, foi criado o protocolo específico de Fisioterapia em Cirurgia Bariátrica e Metabólica.

Os resultados dos dados levantados com os pacientes incorporados atualmente neste protocolo é tema de outro trabalho ${ }^{9}$.

\section{DISCUSSÃO}

Existem poucos sistemas destinados a coletar dados clínicos de uma determinada doença. Centros hospitalares já utilizam bases de dados eletrônicas, porém com destinação às áreas administrativa, operacional, financeira, farmácia, laboratório e radiologia ${ }^{11}$.

Este trabalho busca formatar um "Protocolo Eletrônico" capaz de gerar banco de dados podendo ser de forma multicêntrica e de fácil aplicação, boa qualidade de informações e praticidade de manuseio, mas com característica de não conter excesso de informações (não relevantes), que possam comprometer a praticidade da coleta dos dados.

A realização desta base de dados, e na tentativa de torná-la prática e aplicável às áreas de atuação determinada por este estudo, foi de grande complexidade. A coleta de dados clínicos informatizados além de poupar recursos humanos e financeiros, proporciona redução do tempo na pesquisa ${ }^{1,3,10}$.

O programa prevê recursos que determinam a sua segurança, e uma vez efetuada e finalizada a coleta dos dados, não é possível alterá-los ou editá-los novamente. Mas, a inserção de novos itens é possível, sem alterar o banco de dados já utilizado, ou seja, o aprimoramento e atualização do banco é possível. Os quesitos de segurança citados acima na aplicação do SINPE ${ }^{\odot}$ também se aplicam para o SINPE WEB ${ }^{\odot}$.

Outro aspecto importante com o sigilo e a segurança dos dados, são a necessidade de identificação e a senha de acesso para cada tipo de usuário. Isto dificulta o risco de alterações ou acessos inapropriados e o tipo de permissão outorgada para cada pesquisador.

O aplicativo viabiliza o uso local e remoto, podendo ser executado em máquinas de clientes, servidores de internet e computadores de mão, com a possibilidade de imprimir o protocolo em papel, para realizar a coleta manual, sem perder os parâmetros pré-estabelecidos, em caso de problema de ordem técnica ou elétrica ${ }^{2}$.

Este protocolo possui ainda a característica de permitir a inserção de imagens, vídeos e sons; isto pode ser realizado, facilitando o entendimento pelo examinador, além do benefício de que uma imagem ou vídeo pode ser avaliado por mais de um examinador e servir como referência para avaliar a progressão ou regressão da doença ${ }^{6}$.

\section{CONCLUSÕES}

Com a criação do Protocolo Eletrônico Multiprofissional de Cirurgia Bariátrica e Metabólica, conclui-se que: 1) foi possível criar base eletrônica de dados clínicos referentes à multiprofissionalidade em cirurgia bariátrica e metabólica através da coleta padronizada de informações; 2) foi possível informatizar esta base de dados sob a forma de software; 3) a implantação da base teórica de dados clínicos no protocolo mestre e confecção do protocolo específico foi realizada; 4) a base eletrônica de dados clínicos referentes a multiprofissionalidade em Cirurgia Bariátrica e Metabólica está incorporada ao SINPE ${ }^{\odot}$

\section{REFERÊNCIAS}

1. Blumeinstein BA. Medical research data. Controlled. Clinical Trials 1995; 16:453-455.

2. Brandão FBA. Protocolo Eletrônico de Coletade Dados Clínicos da Isquemia Visceral Crônica. 91f. Tese (Mestrado em Clínica Cirúrgica) - Setor de Ciências da Saúde, Universidade Federal de Paraná, Curitiba, 2009.

3. Davidoff F. Databases in the next millennium. Ann Inter Med. 1997; 117(8):770-774.

4. Koop A, Mösges R. The use of handheld computers in clinical trials. Control Clin Trials 2002; 23:469-480.

5. Lancha JR. Obesidade - Uma Abordagem Multidisciplinar, Rio de janeiro: Ganabara Koogan, 2006.

6. Malafaia O, Kuretzki CH, Schieferdecker MEM, Pinto JSP, Campos ACL, Bortoleto S, Macohin GA, Bortoleto K. Elektronische Datenerfassungim Bereich der klinischen Forschung, Lehreundärztlichen Verwaltung in dem Krankenhaus der Zukunft. 2006.

7. Malafaia O, Borsato EP, Pinto JSP. Gerenciamento do conhecimento em protocolos eletrônicos de coleta de dados. In: simpósio internacional de gestão do conhecimento, 3ed., Curitiba, 2003a.

8. Malafaia O, Borsato EP, Pinto JSP. Manual do Usuário do SINPE, Curitiba: UFPR, 2003b.

9. Nassif DSB, Nassif PAN, Lucas RWC, Ribas-Filho JM, Czeczko NG, KalilFilho FA, Freitas ACT. Efeito da fisioterapia contra-resistida com relação à massa corporal magra em pacientes no pós-operatório de cirurgia bariátrica. ABCD Arq Bras Cir Dig. 2011; 24(3):219-225.

10. RibeiroER. ProtocoloEletrônico deColeta de Dados para Pesquisa em Enfermagem Médico-Cinúrgica. Curitiba. 2004. Dissertação [Mestrado] - Programa de PósGraduação em Clínica Cinúrgica, Universidade Federal Do Paraná.

11. Sigwalt MF. Aplicação multicêntrica informatizada da coleta de dados na doença do refluxo gastroesofágico. [Tese] Universidade Federal do Paraná - UFPR. Curitiba, 2004.

12. Treml CJ. Protocolo eletrônico de coleta de dados clínicos em fisioterapia nas doenças do joelho. 80 f. Dissertação (Mestrado em Clínica Cirúrgica) - Setor de Ciências da Saúde, Universidade federal do Paraná, Curitiba, 2008.

13. Walach VR. Aplicabilidade do Sistema Integrado de Protocolos Eletrônicos SINPE - como ferramenta gerencial na análise de custos do procedimentos da revascularização do miocárdio no Hospital de Clínicas da Universidade Federal do Paraná. 2008. (Mestre). Universidade Federal do Parana, Curitiba. 